\title{
EDITORIAL AND COMMENT Dense Breast Notification Legislation: More Reasons for Caution
}

\author{
Lydia E. Pace, MD, MPH
}

Division of Women's Health, Department of Medicine and Connors Center for Women's Health and Gender Biology , Brigham and Women's Hospital, Boston, MA, USA.

J Gen Intern Med 35(7):1937-9

DOI: $10.1007 / \mathrm{s} 11606-020-05708-2$

(C) Society of General Internal Medicine 2020

$\mathrm{T}$ he care of women with mammographically dense breasts has become one of the most controversial aspects of breast cancer screening. Among women with dense breasts, mammograms have decreased sensitivity for detection of cancer. Women with dense breasts also have a higher risk of developing breast cancer: although data are mixed, one meta-analysis suggested that women with heterogeneously dense (BIRADS category 3 ) breasts have a relative risk of developing breast cancer of 1.6 compared with women with category 2 breast density, while women with extremely dense (category 4) breasts have a relative risk of 2.0. ${ }^{1}$ However, women with dense breasts do not appear to have an increased risk of breast cancer death.

In response to patient advocacy, as of December 2019, 38 states have legislation mandating that patients with increased breast density (or in some states, all women) be notified about their breast density. In 2019, the US Food and Drug Administration (FDA) proposed mandating language for all mammography facilities nationwide to "ensure all women receive consistent breast density information from their mammograms, ${ }^{\text {“2 }}$ whether their density is low or high. For women with high breast density, the FDA's proposed language states: "Your breast tissue density is high. Some patients with high breast density may need other imaging tests in addition to mammograms. Follow the recommendations in this letter, and talk to your healthcare provider about high breast density and how it relates to breast cancer risk, and your individual situation." ${ }^{2}$ The final language is scheduled to be released in September 2020.

Existing and future dense breast notification (DBN) legislation has great relevance for primary care providers (PCPs), since many patients will seek the guidance of their PCPs in response to notification about their breast density. Further, almost half of US women have dense breasts. The challenge facing PCPs and their patients is that there is limited evidence to guide counseling and management of women with dense breasts.

Published online May 28, 2020
In particular, it is not known whether supplemental screening beyond digital mammography improves patient outcomes. MRI and ultrasound increase cancer detection rates but also false positives; whether the increased cancer detection rate improves outcomes (or leads to overdiagnosis) is not known. A recently published randomized controlled trial suggested that supplemental MRI for women with extremely dense (BIRADS category 4) breasts decreased by half the incidence of interval cancers over the 2 years following an initial screen, which could suggest it facilitated earlier diagnosis of cancers that would have become clinically apparent. However, it was also associated with a considerable false positive rate (79.8 per 1000 screenings, versus 16.5 cancers detected per 1000 screenings), and although longer-term follow-up is planned, the study will not be powered to examine impact on breast cancer mortality. ${ }^{3}$ An additional concern with MRI is that although breast MRI without contrast may be routinely performed in the future as the technology advances, in current practice regular breast MRIs require repeated gadolinium administration. The long-term effects of frequent gadolinium exposure are not well-understood but have recently elicited concern because of evidence that some gadolinium is retained in the body long-term. ${ }^{4}$ Digital breast tomosynthesis (DBT) is also being explored as an option for primary or supplemental screening for women with dense breasts. Early data suggest that DBT has better sensitivity and specificity than conventional digital mammography, and especially for women with dense breasts, ${ }^{5}$ but long-term data on outcomes are lacking and this technology is not yet available everywhere.

In light of these uncertainties, some have argued that DBN laws and the FDA's proposed rule are premature. ${ }^{6}$ DBN laws have led to increased utilization of supplemental imaging despite the unclear benefit for patient outcomes. ${ }^{7}$ Some state laws have also been criticized for limited readability of their mandated language, especially women with low literacy, limiting their educational value. ${ }^{8}$ The FDA's proposed language has not undergone the message testing and refinement typically conducted for public health messages. ${ }^{9}$ While we await data to guide care for women with dense breasts, previous and new research on the impact of DBN can help anticipate the impact of the FDA's national rules on patients' experience, service utilization and health care costs, and could inform efforts to optimize their message and implementation. 
The study by Kyanko et al. in this issue of JGIM adds to our understanding of state DBN laws' impact for women. ${ }^{10}$ Kyanko and colleagues use a nationally representative online survey of English-speaking women to examine the association of living in a DBN state with the likelihood that women identified as having dense breasts, their understanding of the clinical implications of dense breasts, and their breast cancer anxiety. Among the 1928 women in their final sample, the authors found that living in a state with DBN laws increased the likelihood of women reporting dense breasts (RR 1.70, 95\% CI $1.34,2.17)$, though a multivariate regression model including an interaction term for educational level and state laws revealed that the laws only had this effect for women who had at least some college education. Among women with lower educational levels, the laws did not increase the likelihood of reporting dense breasts. In adjusted analyses, compared with White women, Hispanic and Black women were less likely to report having dense breasts (OR 0.62 (95\% CI 0.45, 0.85) for Black women, and OR 0.73 (95\% CI $0.55,0.96)$ for Hispanic women). These findings echo another recent survey that found differences in breast density awareness (and anxiety) by race/ethnicity. ${ }^{11}$ However, without further clinical covariates, it is difficult to know the reason for and implications of these racial/ethnic differences in the Kyanko et al. study. The authors also do not examine whether DBN laws had differential impact on awareness for racial/ethnic minority women.

Kyanko et al. find that women in states with DBN laws had increased knowledge of the masking effect of breast density but were not more likely to know about the association between increased breast density and breast cancer risk. Although reporting a history of dense breasts was associated with increased breast cancer anxiety, the study did not find an association between living in a DBN state and increased anxiety. The authors hypothesize that this lack of an association was due to the "small absolute increase in the number of women with anxiety attributable to increased awareness of dense breasts from living in a DBN state."

This study's findings are important for several reasons. First, the study advances the work of prior research on DBN laws' impact by utilizing a nationally representative survey at a time when many states have had these laws in place for some time. Second, consistent with other, smaller studies, this study suggests that DBN laws may not be meeting their intended goal of informing patients of the relationship between breast density and breast cancer risk, a critical aspect of shared decision-making about breast cancer screening and prevention. Third, the authors' examination of the relationship between educational level and the impact of the laws should underscore to the FDA the importance of developing and testing language that will reach the women most at risk of adverse outcomes from breast cancer. Lastly, the study suggests the possibility that the laws may increase breast cancer anxiety while providing limited guidance for counseling, and no strategies shown to actually improve outcomes.

Engaging patients in shared decision-making about breast cancer screening and prevention is a profoundly important goal, and essential for individualizing care. Discussions about breast density could serve as a catalyst for comprehensive breast cancer risk assessment and counseling, and risk assessment tools exist that include breast density along with other risk factors. However, particularly since there is limited understanding of optimal screening strategies among women with dense breasts, messages focused on breast density must be pursued with great care. DBN legislation must be accompanied by a clear plan for implementation that engages both radiologists and PCPs and ideally provides tools to facilitate patient communication about breast cancer risk more broadly, the potential contribution of breast density to that risk, the benefits/risks of screening, and supplemental imaging. Critically, efforts to improve breast cancer outcomes should be particularly focused on women who have completed less education as well as racial/ethnic minority women. As Kyanko et al.'s study demonstrates, current laws run the risk of widening disparities rather than reducing them. Given their impact on millions of women, ongoing research on their effects must be conducted and coupled with advocacy to promote evidence-based and equitable legislation.

Corresponding Author: Lydia E. Pace, MD, MPH; Division of Women's Health, Department of Medicine and Connors Center for Women's Health and Gender Biology Brigham and Women's Hospital, Boston, MA, USA (e-mail: lpace@bwh.harvard.edu).

\section{REFERENCES}

1. Nelson HD, Zakher B, Cantor A, et al. Risk factors for breast cancer for women aged 40 to 49 years: a systematic review and meta-analysis. Ann Intern Med 2012;156(9):635-648.

2. Proposed Rules. Federal Register. 2019;84(60). https://www.govinfo.gov/ content/pkg/FR-2019-03-28/pdf/2019-05803.pdf. Accessed 29 May 2020.

3. Bakker MF, de Lange Sv, Pijnappel RM, et al. Supplemental MRI Screening for Women with Extremely Dense Breast Tissue. N Engl J Med 2019;381(22):2091-2102.

4. Food and Drug Administration. FDA Drug Safety Communication: FDA warns that gadolinium-based contrast agents (GBCAs) are retained in the body; requires new class warnings. 2018; https://www.fda.gov/drugs/ drug-safety-and-availability/fda-drug-safety-communication-fda-warnsgadolinium-based-contrast-agents-gbcas-are-retained-body.

5. Conant EF, Barlow WE, Herschorn SD, et al. Association of Digital Breast Tomosynthesis vs Digital Mammography With Cancer Detection and Recall Rates by Age and Breast Density. JAMA Oncol 2019;5(5):635-642.

6. Keating NL, Pace LE. New Federal Requirements to Inform Patients About Breast Density: Will They Help Patients? Jama. 2019;321(23):2275-2276.

7. Saulsberry L, Pace LE, Keating NL. The Impact of Breast Density Notification Laws on Supplemental Breast Imaging and Breast Biopsy. J Gen Intern Med 2019;34(8):1441-1451. 
8. Kressin NR, Gunn CM, Battaglia TA. Content, Readability, and Understandability of Dense Breast Notifications by State. Jama. 2016;315(16): 1786-1788.

9. The FDA's Opaque Approach To Breast Density Communications. Health Affairs Blog. (December 13, 2019). https://www.healthaffairs.org/do/10. 1377/hblog20191211.729319/full/.

10. Kyanko KA HJ, Busch SH, Aminawung JA, Xu X, Richman IB, Gross CP. Dense breast notification laws, education, and women's awareness and knowledge of breast density: A nationally representative survey. J Gen Intern Med 2020. https://doi.org/10.1007/s11606-019-05590-7.
11. Kressin NR, Wormwood JB, Battaglia TA, Gunn CM. Differences in Breast Density Awareness, Knowledge, and Plans Based on State Legislation Status and Sociodemographic Characteristics. J Gen Intern Med. 2019.

Publisher's Note Springer Nature remains neutral with regard to jurisdictional claims in published maps and institutional affiliations. 